\title{
Uso de métodos multicritérios de tomada de decisão para seleção dos objetivos estratégicos e indicadores de performance considerados no Balanced Scorecard
}

\section{Multi-criteria decision making methods to select strategic objectives and performance indicators considered in the Balanced Scorecard}

\author{
Renata Pelissari Infante \\ Maria Celia de Oliveira' \\ MARIa RItA Pontes AssumpÇãol \\ 'Universidade Metodista de Piracicaba (UNIMEP), Santa Bárbara d'Oeste/SP - Brasil
}

REsumo Balanced Scorecard (BSC) é uma ferramenta utilizada para auxiliar organizações na implementação e acompanhamento de seus indicadores estratégicos. No entanto, o BSC não auxilia na formulação da estratégia. Assim, se os objetivos errados forem estabelecidos, ou as estratégias inadequadas definidas, o BSC será usado para o acompanhamento de aspectos inadequados. Com isso, diversos métodos vêm sendo empregados para auxiliar na formulação da estratégia e na seleção e priorização dos indicadores de desempenho do BSC. O objetivo deste trabalho é identificar quais e como os métodos multicritérios de tomada de decisão, que levam em consideração julgamento e avaliação dos decisores, são utilizados na seleção dos indicadores estratégicos do BSC. Para isso, foi desenvolvida uma revisão sistemática da literatura e identificou-se que AHP é o método multicritério mais utilizado para auxiliar na seleção e priorização dos objetivos estratégicos e indicadores de desempenho usados no BSC. Além disso, técnicas furzy são utilizadas para tratamento dos dados de entrada.

Palavras-chaves: Balanced Scorecard (BSC). Métodos multicritérios, AHP, tomada de DECisÃo, ESTRATÉGia. 
Aвstract Balanced Scorecard (BSC) is a tool used to assist organizations for implementating and monitoring their strategic indicators. However, the does not help in the strategy formulation. Thus, if wrong objectives are set or inappropriate strategies are defined, the BSC will be used to follow up on inappropriate aspects. Several methods have been used to assist the strategy formulation and to help the selection and prioritization of BSC performance indicators. The aim of this work is to identify which and how the multi-criteria decision making methods have been used in the selection of the BSC strategic objectives. For this, we developed a systematic review of the literature. As result, AHP is the most used multi-criteria method to assist in the selection and prioritization of the strategic objectives and performance indicators used in the BSC. In addition, fuzzy techniques are used to treat input data.

Key-words: Balanced Scorecard (BSC). Multi-Criteria decision making methods MCDM, AHP, STRATEGIC MANAGEMENT.

\section{INTRODUÇÃO}

O Balanced Scorecard (BSC), introduzido por Kaplan e Norton em 1991 (KAPLAN E NORTON, 1992), é uma ferramenta utilizada para auxiliar organizações a alcançar seus objetivos estratégicos. O BSC é um mapa estratégico que contribui para a tradução da estratégia da empresa em objetivos estratégicos e indicadores de desempenho, e permite que esses indicadores sejam claramente comunicados a toda a organização e acompanhados ao longo do tempo (KAPLAN e NORTON, 1996; 2000; 2008). Outra contribuição do BSC é permitir a avaliação da contribuição e do impacto das estratégias de diferentes áreas da organização nos resultados da organização como um todo.

Esse método está bastante difundido nas organizações, mas a experiência tem mostrado que sua aplicação não é capaz de, por si só, assegurar uma gestão estratégica bem-sucedida (KAPLAN e NORTON, 2008). O BSC é um mecanismo para implementar a estratégia e não para formulação da estratégia (KAPLAN e NORTON, 1996b). Logo, se os objetivos errados forem estabelecidos, ou as estratégias inadequadas definidas, o BSC será utilizado para o acompanhamento de aspectos inadequados, provendo indicadores de desempenho sem eficácia para avaliar a organização e assegurar sua permanência no mercado.

Com isso, diversos métodos vêm sendo utilizados para auxiliar na formulação da estratégia e na seleção e priorização dos indicadores de desempenho do BSC. Huang, Lai, e Lin (2011) propõem o método multicritério AHP como uma técnica adequada para atribuir pesos e selecionar indicadores de performance do BCS. Por sua vez, Yuan e Chiu (2009) sugerem um sistema de raciocínio ancorado em casos (case-based reasoning-CBR) para auxiliar na atribuição dos pesos em que não há a necessidade da avaliação dos decisores. CBR é um raciocínio máquina que adapta casos semelhantes anteriores para fazer inferências para novos casos.

No entanto, em processos de decisão estratégica, o julgamento e a opinião dos decisores envolvidos são importantes e, por isso, métodos de tomada de decisão que consideram a avaliação dos especialistas são de interesse para esse tipo de situação.

Diante desse contexto, surge o seguinte questionamento: quais e como os métodos multicritérios de tomada de decisão, que consideram o julgamento e avaliação dos decisores, podem auxiliar na atribuição de pesos e na seleção dos objetivos estratégicos do BSC? 
Assim, define-se como objetivo principal deste trabalho o desenvolvimento de uma revisão sistemática da literatura para identificar quais e como os métodos multicritérios de tomada de decisão podem auxiliar na seleção e atribuição de pesos dos objetivos estratégicos do BSC.

Este artigo está organizado como se segue. Na seção 2 é apresentado o referencial teórico. $\mathrm{Na}$ seção 3 são exibidos os procedimentos metodológicos utilizados para realização deste trabalho. Na seção 4 são descritos os resultados da revisão sistemática. E, por fim, na seção 5 são apresentadas as conclusões.

\section{Referencial teÓrico}

Para o entendimento dos resultados obtidos neste trabalho se faz necessário o entendimento de alguns conceitos, que são apresentados nesta seção.

\section{Gestão estratégica e o Balanced Scorecard}

O BSC, desenvolvido por Kaplan e Norton em 1991 (KAPLAN e NORTON, 1992), auxilia no alinhamento entre os objetivos estratégicos de cada área da organização, permitindo a visualização e acompanhamento de indicadores de desempenho que refletem a estratégia da empresa como um todo (KAPLAN e NORTON, 2000). Isso é possível porque o BSC é dividido em quatro perspectivas: financeira, do cliente, dos processos internos e do aprendizado. Cada perspectiva tem seu próprio conjunto de indicadores e esses devem estar conectados de forma a viabilizar o cumprimento dos objetivos estratégicos e da visão da organização (KAPLAN e NORTON, 1996; 2000; 2008).

Existem duas vantagens ao se adotar o BSC para auxiliar na gestão estratégica de uma organização. Primeira, o BSC permite que os gestores vejam, em um único relatório gerencial, o mapa estratégico, diversas informações associadas à estratégia empresarial: indicadores financeiros, indicadores de satisfação do cliente, indicadores de melhoria da qualidade, informações sobre os colaboradores etc. Segunda, permite que os gestores observem como uma melhoria em determinada área pode impactar outra área, positiva ou negativamente, auxiliando na tomada de decisão e no atingimento da estratégia da organização como um todo (KAPLAN e NORTON, 2008).

Os objetivos estratégicos estabelecidos normalmente são derivados de uma visão particular dos gestores e de suas perspectivas de fatores críticos de sucesso, que não necessariamente são as corretas ou melhores. Assim, segundo Kaplan e Norton (2008), mesmo utilizando o melhor BSC, não há garantias de que a organização conseguirá atingir seus objetivos estratégicos. Para isso, é necessário selecionar objetivos estratégicos que realmente representem a visão da organização.

Para auxiliar nesse processo escolha e definição dos pesos dos objetivos estratégicos, pode-se utilizar modelos multicritérios, reduzindo assim, as chances de insucesso na utilização do BSC e, consequentemente, na gestão estratégica. 


\section{Método multicritério de apoio à decisão}

Os métodos multicritérios têm sido desenvolvidos para apoiar e conduzir decisores na avaliação de escolha de alternativas consideradas soluções para determinado problema (GOMES e GOMES, 2014). O conjunto de soluções possíveis é determinado considerando que essas alternativas são as melhores para resolver o problema em questão (VINVKE, 1992).

O resultado pretendido com a utilização dos métodos multicritérios pode ser identificado entre quatro tipos de problemáticas (GOMES e GOMES, 2014):

- Procedimento de seleção ou escolha de uma alternativa;

- Classificação das alternativas;

- Ordenação das alternativas;

- Esclarecer a decisão por uma descrição.

Os principais métodos multicritérios de tomada de decisão (MDCM), segundo Leite e Freitas (2012), são: Analytic Hierarchy Process (AHP) (SAATY, 1987), Elimination and Choice Expressing Reality (ELECTRE) (ROY, 1996) e Preference Ranking Organization Method for Enrichment Evaluations (PROMETHEE) (BRANS e VINCKE, 1985).

Uma característica importante dos métodos multicritérios em relação a outros métodos de tomada de decisão é a inclusão do julgamento e avaliação dos decisores no processo de decisão.

\section{Procedimentos metodológicos}

A Revisão Sistemática da Literatura é um instrumento usado para mapear trabalhos publicados sobre um tema específico de pesquisa, permitindo ao pesquisador elaborar uma síntese do conhecimento existente a respeito do assunto (BIOLCHINI et al., 2007) e, utilizando-se dessa síntese, responder a uma pergunta de pesquisa (CORDEIRO et al., 2007).

Para o desenvolvimento deste trabalho, foi adotado um processo de revisão sistemática da literatura ancorado no método proposto por Biolchinni et al. (2005), que possui três etapas: planejamento da revisão, execução da revisão e análise dos resultados. Os detalhes desse processo são apresentados no Quadro 1.

\section{Desenvolvimento}

A base de pesquisa escolhida para a busca de documentos foi a Scopus. A string de busca adotada foi AHP ou ANP ou ELECTRE ou PROMETHÈ ou MULTICRITERIA ou "MULTI-CRITERIA" ou "MULTIPLE-CRITERIA" ou MCDM e BSC ou "BALANCEND SCORECARD”, utilizadas nos critérios de busca em título, resumo e palavras-chave. Foram encontradas 182 publicações.

Em seguida, foram aplicados os seguintes filtros de seleção: tipo de documento - artigo e idioma - inglês, resultando em 109 artigos selecionados. Foram excluídos os artigos de áreas de pesquisa não alinhadas ao objetivo deste estudo, como Psicologia, Medicina, Artes, entre outras, restando 101 artigos. 
Quadro 1 - Processo de execução da revisão sistemática de literatura utilizada neste artigo.

\section{Planejamento da revisão}

\section{Definição da questão de pesquisa e criação do protocolo de pesquisa. $\mathrm{O}$ protocolo de pesquisa contempla:}

- Definição do objetivo da revisão

- Escolha das bases de pesquisa

- Definição dos filtros e critérios de seleção

\section{Execução da revisão}

Busca dos documentos e aplicação dos filtros e critérios de seleção, definidos na etapa de planejamento.

\section{Análise dos resultados}

Leitura, síntese e análise dos documentos selecionados, respondendo a questão de pesquisa

Fonte: Adaptado de Biolchinni et al. (2005).

Informações dos 101 artigos selecionados, como título, autores, ano de publicação, número de citações, entre outras, foram exportadas da base do $S$ copus em um arquivo txt e importados para o software $\mathrm{R}$, onde foram aplicados outros filtros de seleção. $\mathrm{R}$ é uma linguagem e uma suíte integrada de instalações de software para manipulação de dados, cálculo e exibição gráfica (R-PROJECT, 2016). A codificação em código R foi utilizada por permitir a automatização da aplicação de filtros, diminuindo as chances de erros resultantes de manipulações manuais por parte do pesquisador.

No R, primeiramente foi aplicado um filtro de JCR, selecionando-se apenas artigos de periódicos com JCR. O JCR (Journal Citation Reports) é um indicador que possibilita avaliar criticamente a influência das revistas mais importantes do mundo, com informações quantificáveis, e é um valor calculado com base em dados de citação. Quanto maior o indicador JCR, tanto maior a influência e a importância da revista e, consequentemente, mais relevantes são suas publicações. 
A aplicação do filtro de JCR resultou na seleção de 41 artigos, posteriormente separados em recentes e antigos, considerando como recentes os artigos publicados a partir de 2014. Dos 39 artigos, 15 são recentes e 26 antigos.

Para artigos identificados como antigos, foi aplicado um filtro de seleção conforme seu reconhecimento científico, utilizando como medida a média de citações por ano desde sua publicação. Foram selecionados apenas os artigos antigos com uma média de pelo menos duas citações por ano, o que resultou na seleção de 20 artigos. Esse filtro não foi aplicado para os artigos recentes, pois esses não tiveram tempo suficiente de serem citados. Foram selecionados um total de 35 artigos.

Em seguida, foram lidos os títulos e resumos dos 35 artigos e foram selecionados apenas os que atendem aos critérios de inclusão, que sejam:

- Utilizar ou sugerir a utilização do BSC;

- Utilizar métodos multicritérios para atribuir pesos e selecionar objetivos estratégicos utilizados no BSC;

- Estar disponível na íntegra.

Desses, oito artigos não estão disponíveis na íntegra e cinco não estão alinhados com o objetivo da pesquisa, restando 22 artigos, que foram lidos na íntegra. Desses, 14 artigos foram desconsiderados pois, apesar de utilizarem BSC e modelos de tomada de decisão multicritério, não propõem soluções para a seleção de indicadores, interesse desta pesquisa. Os oito artigos restantes foram analisados e sintetizados, com o intuito de responder à questão de pesquisa deste estudo. Os resultados encontrados são apresentados na seção a seguir.

\section{Resultados}

Os artigos selecionados, apresentados no Quadro 2, foram lidos na íntegra e analisados com o objetivo de identificar quais e como métodos multicritérios são utilizados para auxiliar na seleção dos objetivos estratégicos considerados no BSC.

No que diz respeito às áreas de aplicação, constatou-se que há uma diversidade de setores estudados: telecomunicação, farmacêutica, manufatura, sustentabilidade, finanças e engenharia civil.

O principal periódico é o Expert Systems with Applications que contém cinco dos oito artigos selecionados.

Após a leitura completa dos artigos, identificou-se que seis usam o método multicritério Analytic Hierarchy Process (AHP) ou uma variação do AHP, o Analytic Network Process (ANP), para selecionar e atribuir pesos aos objetivos estratégicos do BSC, um estudo usa o AHP para atribuir pesos e o VIKOR para selecionar os objetivos e um estudo utiliza o método SMARTER.

O método SMARTER (Simple Multiattribute Rate Technique Exploiting Ranks) foi desenvolvido por Edwards e Barron (1994) e é adequado para a priorização de alternativas. O método VIKOR é utilizado para priorizar e selecionar alternativas em problemas de decisão com critérios conflitantes (OPRICOVIC e TZENG, 2004). O método AHP, por ser o método mais usado nos estudos selecionados, será descrito em detalhes na seção a seguir. 
Quadro 2 - Informação dos artigos selecionados

\begin{tabular}{|c|c|c|c|c|c|c|c|c|}
\hline 量 & 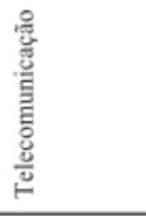 & 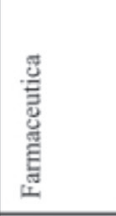 & 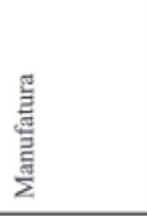 & . & 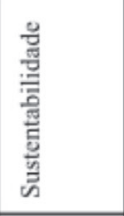 & 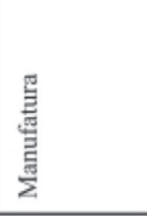 & 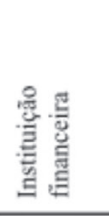 & 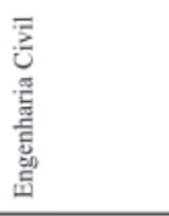 \\
\hline 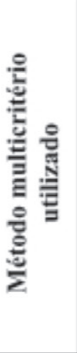 & 全 & 裹 & $\hat{\mathrm{z}}$ & 全 & 全 & 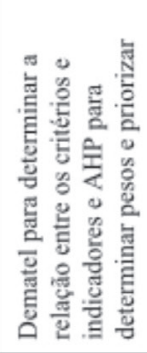 & 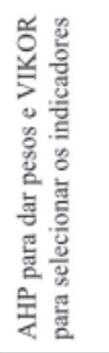 & 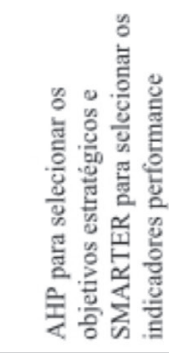 \\
\hline 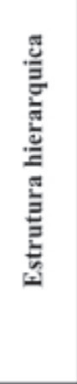 & 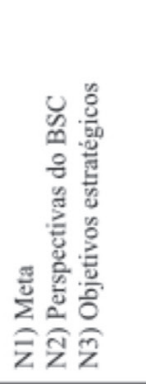 & 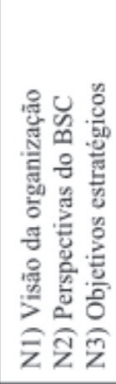 & 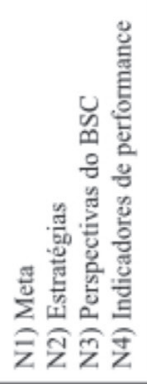 & 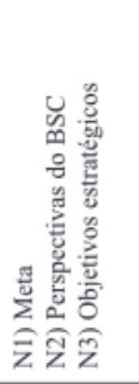 & 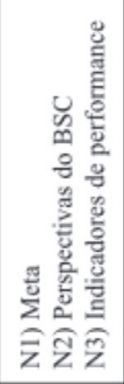 & 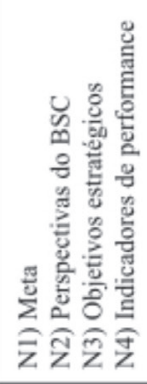 & 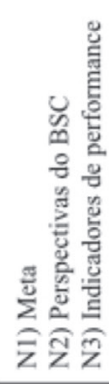 & 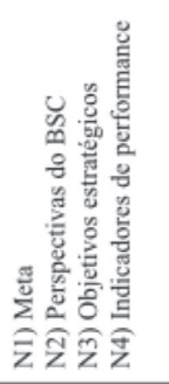 \\
\hline 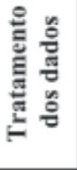 & ' & ' & $\frac{1}{3}$ & ' & 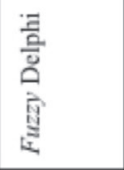 & ' & 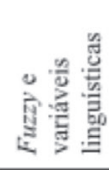 & ' \\
\hline 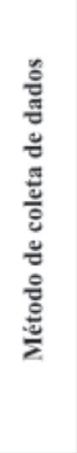 & 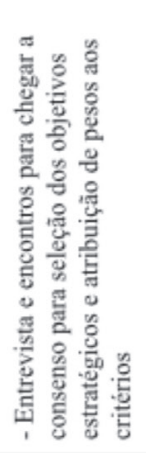 & 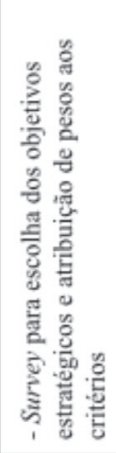 & 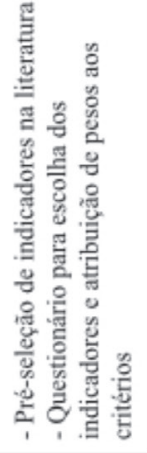 & 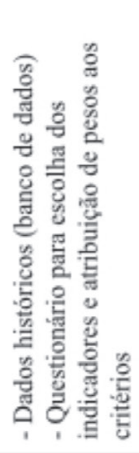 & 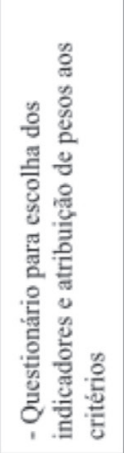 & 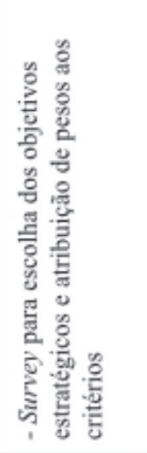 & 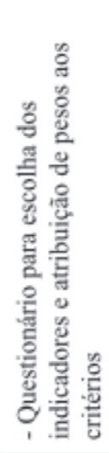 & ' \\
\hline 竞 & 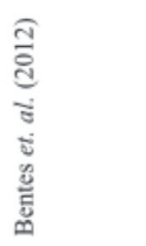 & 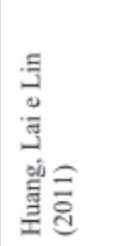 & 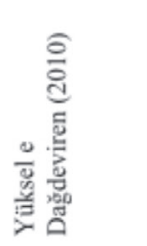 & 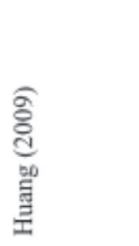 & 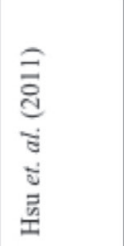 & 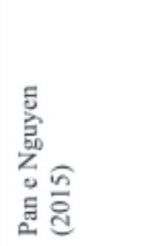 & 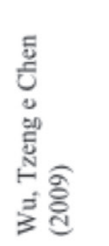 & 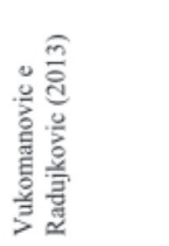 \\
\hline
\end{tabular}




\section{Os MÉTOdos AHP E ANP}

O método AHP foi desenvolvido por Saaty (1987), e é um processo de tomada de decisão para a definição de prioridades e seleção de alternativas. Esse método permite obter o entendimento de decisões complexas pela decomposição do problema em uma estrutura hierárquica.

Assim, ao utilizar o método AHP, o problema de decisão é estruturado em diversos níveis. O primeiro nível é o objetivo ou meta que direciona a tomada de decisão. O segundo nível é formado por critérios de decisão, o terceiro por subcritérios desses critérios e assim por diante, até o último nível que contém as alternativas de decisão. Essa relação hierárquica é apresentada na Figura 1.

Os decisores avaliam os critérios comparativamente de forma a atribuir pesos. Com isso, o método AHP reconhece explicitamente e incorpora o julgamento e a experiência dos decisores no processo de definição de prioridades.

O ANP, também introduzido por Saaty, é uma generalização do AHP (SAATY, 2005). Enquanto o AHP representa uma estrutura com uma relação hierárquica unidirecional, o ANP permite inter-relações entre níveis de decisão e entre alternativas. Por exemplo, não só a importância dos critérios determina a importância das alternativas, como em uma hierarquia, mas também a importância das alternativas podem ter impacto sobre a importância dos critérios. Com isso, o ANP é adequado para situações em que os critérios não são independentes.

Figura 1 - Exemplo da estrutura hierárquica do modelo AHP

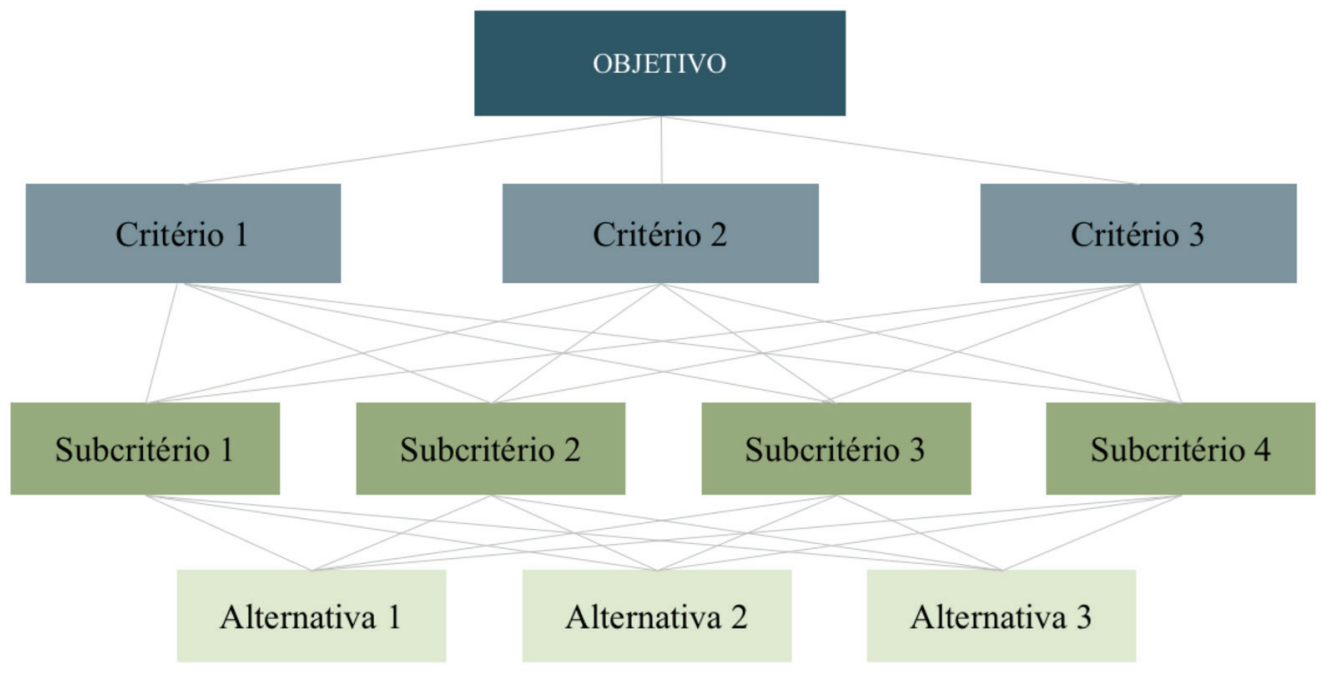

\section{Métodos utilizados para a atribuição de pesos e seleção de indicado- res do BSC}

A relação hierárquica do AHP/ANP utilizada nos estudos selecionados foi estruturada considerando a visão da organização ou uma meta específica como direcionador, as perspectivas do BSC (financeira, cliente, processos internos e aprendizado) como critérios e os objetivos estratégicos ou indicadores de desempenho como alternativas de decisão. A Figura 2 apresenta um exemplo dessa estrutura. 
Figura 2 - Exemplo de estrutura do modelo AHP utilizado para a atribuição de peso e seleção dos objetivos estratégicos do BSC

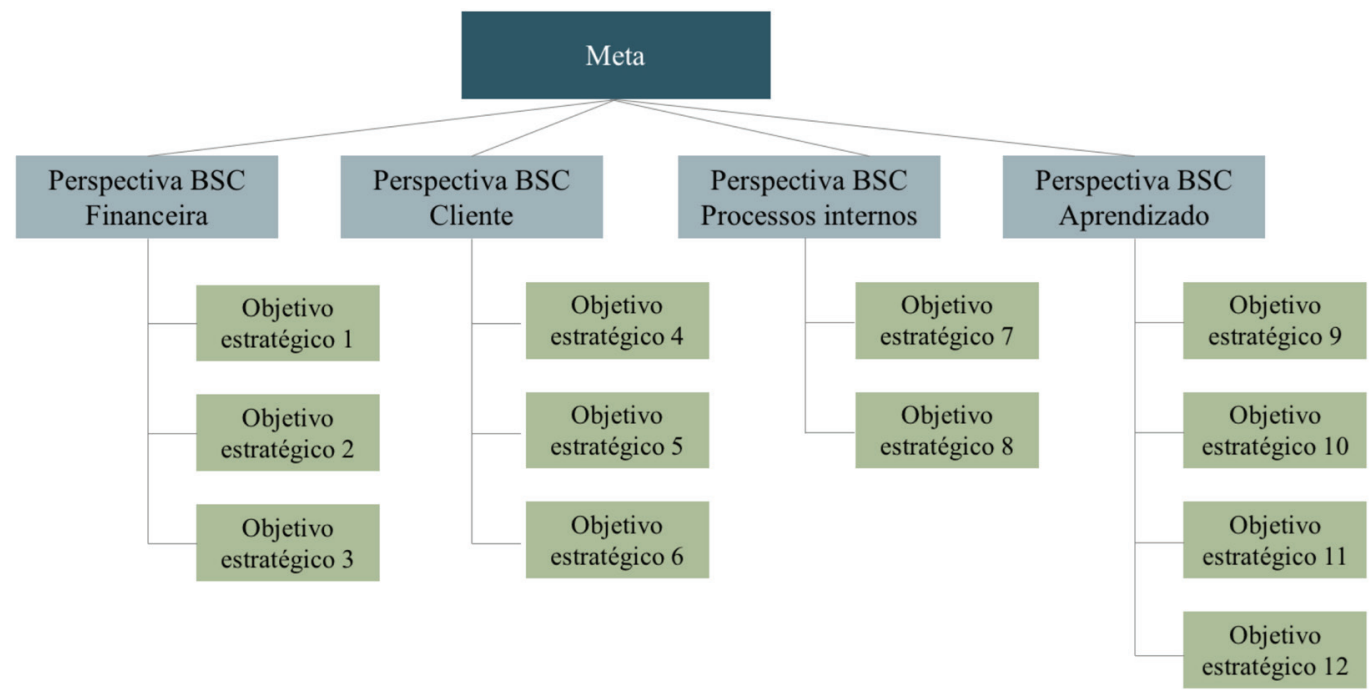

Apesar da similaridade entre os estudos na forma de estruturar o problema de decisão em níveis, algumas particularidades foram identificadas. Essas particularidades dizem respeito à: (i) quantidade de níveis da hierarquia; (ii) forma de se obter os dados de entrada; (iii) tratamento dos dados de entrada (iv) método utilizado para a seleção dos indicadores. Cada uma dessas particularidades será discutida a seguir.

Em relação à quantidade de níveis de hierarquia, a maioria dos estudos utilizou três níveis, de forma similar ao apresentado na Figura 2 (HUANG, LAI e LIN, 2011; BENTES et al., 2012; HUANG 2009; WU, TZENG e CHEN, 2009; HSU et al., 2011). Diferentemente, Yüksel e Dağdeviren (2010) e Pan e Nguyen (2015) adotaram uma estrutura hierárquica com quatro níveis. A estrutura usada por Yüksel e Dağdeviren (2010) considera a visão da organização como direcionador, as estratégias da organização como critérios, as perspectivas do BSC como subcritérios e os indicadores de desempenho como alternativas de decisão. Já a estrutura empregada por Pan e Nguyen (2015) considera a visão da organização como direcionador, as perspectivas do BSC como critérios, os objetivos estratégicos como subcritérios e os indicadores de desempenho como alternativas de decisão.

Em relação aos dados de entrada, tem-se que as informações necessárias para a aplicação do método AHP são: lista dos objetivos estratégicos a serem considerados como alternativas e a avaliação dos especialistas para a definição dos pesos dos critérios. Huang, Lai e Lin (2011), que propõem a utilização do modelo AHP para priorizar os objetivos estratégicos considerados no BSC de empresas farmacêuticas, utilizaram uma survey com 50 respondentes de diversas empresas farmacêuticas de Taiwan para obter as informações de entrada. Pan e Nguyen (2015) também usaram uma survey para a coleta dos dados de entrada. Por sua vez, Bentes et al. (2012) obtiveram as informações de entrada entrevistando conjuntamente todos os especialistas em cinco encontros programados. 
As informações de entrada também podem ser obtidas por meio da aplicação de questionários conforme utilizado por Hsu et al. (2011) e Huang (2009). Wu, Tzeng e Chen (2009) e Yüksel e Dağdeviren (2010), também utilizaram questionários. No entanto, antes da coleta de dados, indicadores de desempenho foram pré-selecionados por meio de uma revisão sistemática. Só então questionários foram aplicados ao especialista para a escolha dos indicadores entre os pré-selecionados e para atribuição de pesos aos critérios.

Em alguns estudos, os dados de entrada contendo as avaliações dos especialistas foram tratados antes de serem utilizados no método multicritério. Wu, Tzeng e Chen (2009) e Yüksel e Dağdeviren (2010) empregaram a teoria furzy com variáveis linguísticas e Hsu et al. (2008) usaram método furzyy Delphi. O primeiro caso é adequado quando as avaliações dos especialistas ao comparar critérios são dadas por expressões, por exemplo, "mais importante", "menos importante". Ao se usar variáveis linguísticas e teoria fuzzy é possível quantificar as avaliações e reduzir a incerteza presente nesse tipo de avaliação. O método fu₹:y Delphi é uma técnica aplicada em situações de decisão em grupo com o objetivo de se obter consenso entre as avalições dos especialistas. Para empregar essa técnica, é necessária a aplicação de questionários repetidas vezes, intercalados com seções de feedback.

Em relação aos métodos multicritérios empregados para a atribuição de pesos e seleção dos indicadores, quatro artigos utilizaram apenas o método AHP (BENTES et al. [2012], HUANG, LAI e LIN, 2011; HUANG, 2009 e HSU et al., 2011).

Yükse (2010) utilizou o ANP, pois considera que os critérios “perspectivas do BSC" não são independentes, como requer o método AHP. Portanto, o ANP seria uma técnica mais adequada para essas situações de dependência entre os critérios.

Huang (2009) também utiliza o AHP, mas propõe a integração do BSC com os Sistemas Baseados em Conhecimento - Knowledge Based Systems (KBS) resultando na proposta de um modelo chamado intellectual BSC knowledge-based system (BSCKBS). A diferença, nesse caso, é o uso de um sistema que automatiza o processo de seleção dos indicadores de desempenho e de atribuição de pesos. O questionário para obter as informações de entrada é aplicado via web e informações históricas presentes em banco de dados também podem ser utilizadas no modelo AHP. A aplicação do método AHP é realizada de forma automática. Com isso, o BSCKBS possibilita a rápida implementação de planos estratégicos e ajuda os clientes a serem mais eficientes na execução dos planos estratégicos.

De forma geral, os resultados obtidos nesses estudos que utilizam o método AHP ou o método ANP, são: (i) pesos de importância de cada uma das perspectivas do BSC, revelando a perspectiva mais importante; (ii) pesos das alternativas (objetivos estratégicos ou indicadores estratégicos) dentro de cada uma das perspectivas; (iii) pesos globais das alternativas, que representam a importância de cada alternativa em relação à meta; e, finalmente (iv) com os pesos globais é possível selecionar ou priorizar as alternativas mais importantes para atingir a meta estabelecida.

Wu, Tzeng e Chen (2009) também utilizam o método AHP para dar pesos aos indicadores de desempenho, mas usam técnica multicritério VIKOR para selecionar os indicadores de desempenho, baseando-se nos pesos já definidos. 
O estudo desenvolvido por Vukomanovic e Radujkovic (2013) propõe a conexão do BSC com outro modelo de gestão, o Excellence Model (EFQM), além de utilizar uma estrutura diferente da apresentada na Figura 2. O EFQM é baseado nos princípios do Total Quality Management (TQM) e é um modelo que pode ser usado para verificar o alinhamento da estratégia com o meio ambiente, avaliação não possível com o BSC.

A metodologia utilizada para conectar esses dois modelos foi: (b) definição dos objetivos estratégicos, do mapa do BSC e do EFQM (a) utilização do AHP para dar pesos aos objetivos estratégicos considerando como critérios do modelo AHP os critérios do EFQM. Em seguida, os objetivos com menos peso foram descartados do mapa inicial e (c) para cada objetivo estratégico restante no mapa do BSC, indicadores de desempenho foram escolhidos usando o critério SMARTER.

Resumidamente, o trabalho de Vukomanovic e Radujkovic (2013) propõe um modelo que utiliza o AHP e os critérios do EFQM para a seleção dos objetivos estratégicos e usa o método SMARTER para a seleção dos indicadores de desempenho do BSC.

Os resultados obtidos nos estudos aqui analisados foram considerados satisfatórios e as perspectivas do BSC ou os indicadores considerados como os mais importantes podem servir como diretrizes e referências para organizações do mesmo ramo (HSU et al., 2011; HUANG, LAI e LI, 2011; WU, TZENG e CHEN, 2009; PAN e NGUYEN, 2015).

\section{ConsideraçÕES FINAIS}

Este artigo apresentou uma revisão da literatura sobre a aplicação de métodos multicritério para selecionar e priorizar objetivos estratégicos e indicadores de desempenho utilizados no BSC. Ao todo, 182 artigos foram encontrados na busca inicial e nove foram selecionados após a aplicação das regras de seleção e inclusão.

O AHP é o método multicritério mais empregado para auxiliar na seleção e priorização dos objetivos estratégicos e indicadores de desempenho usados no BSC e técnicas furzy são utilizadas para tratamento dos dados de entrada.

Um estudo utilizou o método SMARTER e um estudo empregou o método VIKOR para a seleção dos indicadores após determinar os pesos dos indicadores pela aplicação do método AHP.

Um estudo utilizou o método Dematel para determinar a relação entre os critérios, entre os indicadores e entre os critérios e indicadores, antes da aplicação do AHP para a atribuição dos pesos de importância.

\section{ReFERÊNCIAS}

BENTES; A. V.; CARNEIRO; J.; DA SILVA; J. F.; KIMURA; H. "Multidimensional assessment of organizational performance: Integrating BSC and AHP.” Journal of Business Research, v. 65, p. 1.790-1.799, 2012. 
BIOLCHINNI, J.; MIAN, P. M.; NATALI, A. C. C.; TRAVASSOS, G. H. "Systematic review in software engineering" (Report). Rio de Janeiro: 2005. Disponível em <http://www.cin. ufpe.br/ in1037/leitura/systematicReviewSE-COPPE.pdf $>$. Acessado em 26 de janeiro de 2016.

BRANS, J. P.; VINCKE, P. H. "A preference ranking organization method, the PROMETHEE method for MCDM.” Management Science, v. 31, p. 647-656, 1985.

CHEN, T. L; LIN, K. L. "Complementing AHP with habitual domains theory to identify key performance indicators for service industry." IEEE International Conference on Service Operations and Logistics, and Informatics, p. 84-89, 2006.

CONFORTO, E. C.; AMARAL, D. C.; SILVA, S. L. "Roteiro para revisão bibliográfica sistemática: aplicação no desenvolvimento de produtos e gerenciamento de projetos." CBGDP, 2011.

CORDEIRO, A. M.; OLIVEIRA, G. M.; RENTERÍA, J. M.; GUIMARÃES, C. A. "Revisão sistemática: uma revisão narrativa.” Rev. Col. Bras. Cir., v. 34, p. 428-431, 2007.

EDWARDS, W.; BARRON, F. H. "SMARTS and SMARTER: Improved simple methods for multiattribute utility measurements." Organizational Behavior and Human Decision Processes, v. 60, p. 306-325, 1994.

GOMES, L. F. A. M.; GOMES, C. F. S.; ALMEIDA, A. T. de. Tomada de decisão gerencial: o enfoque multicritério, 5. ed. Rio de Janeiro: Ed. Atlas, 2014.

HSU; C.-W.; HU; A. H.; CHIOU; C.-Y.; CHEN; T.-C. "Using the FDM and ANP to construct a sustainability balanced scorecard for the semiconductor industry." Expert Systems with Applications, v. 38, p. 12.891-12.899, 2011.

HUANG, H. "Designing a knowledge-based system for strategic planning: A balanced scorecard perspective.” Expert Systems with Applications, v. 36, p. 209-218, 2009.

HUANG, H.; LAI, M.; LIN, L. "Developing strategic measurement and improvement for the biopharmaceutical firm: Using the BSC hierarchy.” Expert Systems with Applications, v. 38, p. 4.875-4.881, 2011.

KAPLAN, R.; NORTON, D. "The Balanced Scorecard - Measures that drive performance." Harvard Business Review, v. 70, p. 71-79, 1992.

KAPLAN, R.; NORTON, D. "Using the balanced scorecard as a strategic management system.” Harvard Business Review, v. 74, p. 75-85, 1996.

KAPLAN, R.; NORTON, D. "Strategic learning and the balanced scorecard." Strategy and Leadership, v. 24, p. 19-24, 1996.

KAPLAN, R.; NORTON, D. “Having Trouble With Your Strategy? Then map it.” Harvard Business Review, v. 78, p. 167-176, 2000. 
KAPLAN, R.; NORTON, D. "Mastering the Management System." Harvard Business Review, v. 86, p. 62-77, 2008.

LEITE, I. M. S.; FREITAS, F. F. T. “Análise Comparativa dos métodos de apoio multicritério a decisão: AHP, ELECTRE e PROMETHEE.” XXXII Encontro Nacional de Engenharia de Produção, 2012.

OPRICOVIC, S.; TZENG, G. "Compromisse solution by MCDM methods: a comparative analysis of VIKOR and TOPSIS.” European Journal of Operational Research, v. 16, p. 445-455, 2004.

PAN; J.-N.; NGUYEN; H. T. N. "Achieving customer satisfaction through product-service systems.” European Journal of Operational Research, v. 247, p. 179-190, 2015.

ROY, B. Multicriteria Methodology Goes Decision Aiding. Netherlands: Kluwer Academic Publishers, 1996.

R-project. Disponível em: < https://www.r-project.org/about.html>. Acessado em 20 janeiro de 2016.

SAATY, T. L. "The analytic hierarchy process - what it is and how it is used." Mathematical Modelling, v. 9, p. 161-176, 1987.

SAATY, T. L. Theory and Applications of the Analytic Network Process. Pittsburgh, PA: RWS Publications, 2005.

VUKOMANOVIC, M.; RADUJKOVIC, M. "The balanced scorecard and EFQM working together in a performance management framework in construction industry." Journal of Civil Engineering and Management, v. 19, p. 683-695, 2013.

WU; H.-Y.; TZENG; G.-H.; CHEN; Y.-H. “A fuzzy MCDM approach for evaluating banking performance based on Balanced Scorecard.” Expert Systems with Applications, v. 36, p. 10.135-10.147, 2009.

YUAN; F.-C.; CHIU; C. "A hierarchical design of case-based reasoning in the balanced scorecard application.” Expert Systems with Applications, v. 36, p. 333-342, 2009.

YÜKSEL; I.; DAĞDEVIREN; M. "Using the fuzzy analytic network process (ANP) for Balanced Scorecard (BSC): A case study for a manufacturing firm.” Expert Systems with Applications, v. 37, p. 1.270-1.278, 2010.

\section{Dados das Autoras}

\section{Renata Pelissari Infante}

Doutoranda em Engenharia de Produção pela Universidade Metodista de Piracicaba. Santa Bárbara d'Oeste/SP - Brasil. renatapelissari@gmail.com 


\section{Maria Celia de Oliveira}

Doutora em Engenharia de Produção pela Universidade Metodista de Piracicaba. Santa Bárbara d'Oeste/SP - Brasil. marolivei@unimep.br

\section{Maria Rita Pontes Assumpção}

Doutora em Engenharia de Produção pela Universidade de São Paulo. Santa Bárbara d’Oeste/ SP-Brasil.mrpontes@unimep.br

Submetido em: 08-12-2016

Aceito em: 28-03-2017 